\title{
Estudo dos sinais de DTM em pacientes ortodônticos assintomáticos*
}

\author{
Maria Eloiza G. Delboni**, Jorge Abrão***
}

\begin{abstract}
Resumo
O objetivo deste trabalho foi estudar a presença de sinais de disfunção têmporo-mandibular em pacientes assintomáticos ao início, durante e após o tratamento ortodôntico, através de uma revisão da literatura, verificando se o exame clínico é um método suficiente para diagnóstico, e se o tratamento ortodôntico é fator contribuinte para o desenvolvimento dessas disfunções têmporo-mandibulares nestes pacientes. $\mathrm{O}$ tratamento ortodôntico não pode ser considerado fator contribuinte para desenvolvimento de disfunções têmporo-mandibulares.
\end{abstract}

Palavras-chave: Têmporo-mandibular. Ortodontia. Ortodontia corretiva. Síndrome da disfunção têmporo-mandibular. Oclusão. Má oclusão.

\section{INTRODUÇÃO}

A prevalência de sinais assintomáticos e sintomáticos relativos às disfunções têmporo-mandibulares tem aumentado significantemente nas últimas décadas. Há muitas controvérsias relacionadas à etiologia dessas desordens, sendo preponderante a que relaciona a multiplicidade de fatores como hiperatividade muscular, trauma, estresse emocional, má oclusão, além de inúmeros outros fatores predisponentes, precipitantes ou perpetuantes dessa condição ${ }^{22}$. Em virtude da complexidade etiológica e da variedade dos sinais e sintomas que podem, genericamente, também representar outras patologias, o reconhecimento e a diferenciação das desordens têmporo-mandibulares podem apresentar-se de forma não muito clara ao profissional. Portanto, é importante realizar, além do exame de rotina, uma anamnese direcionada e exame clínico seletivo, para que em conjugação com o conhecimento específico do profissional levem ao diagnóstico e, subseqüentemente, à elaboração do plano de tratamento.

\section{REVISÃO DE LITERATURA}

Através da revisão de literatura, Perry ${ }^{23}$ estudou a disfunção têmporo-mandibular em adolescentes pré-ortodônticos, observando que muitos adolescentes apresentavam sintomas de DTM nesta fase em que estavam sendo sempre vistos pelo ortodontista, o qual tinha a responsabilidade com a oclusão funcional equilibrada, e que muitos ortodontistas negligenciavam a integridade das articulações têmporo-mandibulares e da musculatura mandibular.

Helkimo 6 enfatizou a necessidade de uniformizar as pesquisas nessa área, para esclarecer aspectos ainda obscuros das desordens da articulação têmporo-mandibular. Relatou que os sintomas são

*Resumo da tese de mestrado apresentada à Escola Paulista de Medicina - UNIFESP

** Ortodontista, Especialista pela USP-Bauru, Mestranda em Morfologia aplicada à Área da Saúde pela EPM-UNIFESP.

*** Professor Livre Docente do Departamento de Ortodontia e Odontopediatria, Disciplina Ortodontia da Universidade de São Paulo-USP. 
muito comuns em uma população aleatória e afirmou que não existem diferenças entre os gêneros em relação à prevalência de sintomas. Ressaltou, ainda, o caráter multifatorial da etiologia das desordens do sistema mastigatório.

Aubrey $^{1}$, ao estudar as causas da disfunção da articulação têmporo-mandibular em pacientes adolescentes durante o período de tratamento ortodôntico, assegura que a disfunção não se manifesta durante os anos que o profissional mantém o paciente sob observação, por isso o jovem pode tolerar a disfunção durante anos, antes de algo provocar os característicos espasmos e sintomas desta sindrome. No entanto alguns destes jovens pacientes têm estes sintomas, mas nunca os menciona ao ortodontista porque não associam os dentes às dores de cabeça.

Sadowsky e Begole ${ }^{28}$ realizaram um estudo comparando 75 pacientes que tinham se submetido a tratamento ortodôntico quando adolescentes, com uma amostra de 75 pacientes que nunca receberam tratamento ortodôntico. Os autores avaliaram a oclusão funcional com a função da articulação têmporo-mandibular e encontraram bastante similaridade na prevalência de sinais e sintomas nas articulações têmporo-mandibulares, entre os grupos.

Combinando artrografia e cineradiografia, Isberg e Westesson ${ }^{9}$ estudaram o movimento do disco e côndilo na articulação têmporo-mandibular com e sem estalidos (clicking), dissecando a autópsia de cinco amostras da ATM com estalidos e quatro sem estalidos (clicking). Concluíram que os estalidos (clicking) estavam associados a irregularidades na eminência articular durante o percurso condilar.

Willianson ${ }^{37}$ relatou a importância de se realizar um exame clínico criterioso de sinais e sintomas de DTM, antes de se iniciar o tratamento ortodôntico, para que o paciente seja alertado quando da presença desses sinais e/ou sintomas. Nos casos em que se detecta a presença de estalidos, deve-se tentar recapturar o disco antes de iniciar o tratamento, e quando o paciente já se en- contra com travamento de abertura bucal, indicase o tratamento para estabilizar a oclusão e aliviar a dor, antes de procedimentos irreversíveis.

Sadowsky e Polson ${ }^{29}$, a fim de investigarem as desordens têmporo-mandibulares e a oclusão funcional após o tratamento ortodôntico, realizaram dois estudos longitudinais. Foram selecionados dois grupos de pacientes com más oclusões de Classes I, II ou III, tratados ortodonticamente e outro grupo não tratados, pelo menos há 10 anos. Quanto à oclusão funcional, verificou-se uma alta incidência de contatos em balanceio, em todos os grupos, não havendo diferença estatística entre eles. Os autores concluíram que as correções ortodônticas com aparelho fixos, na adolescência, não aumentam e nem diminuem, em geral, o risco de que se desenvolvam desordens têmporo-mandibulares no futuro.

Ao estudarem os sons da ATM com a morfologia da articulação, Rohlin et al. ${ }^{27}$ observaram que $58 \%$ das articulações eram silenciosas, 20\% apresentavam estalidos e um deslocamento anterior de disco. Outros 22\% apresentavam crepitação e exibiam, em sua maioria, artrose das superfícies articulares e perfuração dos discos. Estes resultados confirmam que estalidos e crepitações podem estar correlacionados como sinais de morfologia anormal das articulações. Mas a ausência de som não deve ser fator indicativo de articulação normal.

Sadowsky et al. ${ }^{31}$ examinaram 98 pacientes pré-ortodônticos, 176 durante o tratamento ativo e 73 pós-tratamento, estabelecendo a prevalência de ruídos articulares e sua possível relação com a Ortodontia. A prevalência para o grupo pré-tratamento foi de $40,8 \%$, sendo de $60,8 \%$ para o grupo em tratamento e $68,5 \%$ para os jovens já tratados. Concluíram que os sons provenientes da ATM são muito comuns antes, durante e depois do tratamento ortodôntico.

Ao estudarem a correlação dos sons da articulação têmporo-mandibular com a morfologia da ATM, Eriksson et al. ${ }^{3}$ afirmaram que os sons podem ser vistos como sinais de diferentes tipos de 
patologias. No entanto, o estalido e a crepitação não indicam que é uma articulação com morfologia anormal. O estalido (clicking) duplo, na abertura e fechamento mandibular, caracteriza-se por deslocamento de disco articular com redução, e a articulação silenciosa assintomática e limitação na abertura indicam deslocamento de disco articular sem redução. A crepitação freqüentemente indica uma artrose. Concluíram que o grau de deformação e deslocamento articular não podem ser analisados através dos sons. Entretanto, a ausência de sons não deve ser aceita como indicação de ATM normal.

Por meio de imagem de ressonância magnética, Kircos et al. ${ }^{13}$ examinaram 42 articulações têmporo-mandibulares. Demonstraram que a presença de deslocamento de disco (32\% da amostra) não indica, necessariamente, a existência de DTM. Apesar disso, os autores não excluem a possibilidade de haver pacientes assintomáticos que futuramente venham a manifestar disfunção.

Kaplan et al. ${ }^{11}$, em um estudo sobre articulações têmporo-mandibulares, concluiram que existe uma variabilidade na configuração e na espessura de um disco articular normal e que muitas vezes uma posição superior e anterior do disco articular não representa estado patológico de um deslocamento do disco articular.

O estalido, segundo Pullinger et al..$^{25}$, é o mais comum dos ruídos e representa um desequilíbrio mecânico da articulação têmporo-mandibular e dos músculos da mastigação.

Westesson et al..$^{35}$ examinaram 40 pacientes assintomáticos através de exames de artrografia e radiografia, com ATM clinicamente normal, para verificarem o grau de confiabilidade do exame clínico. Os resultados mostraram (85\%) das articulações normais e (15\%) destes pacientes assintomáticos tinham alguma forma de deslocamento, o que pode sugerir que exame clínico negativo das articulações pode envolver um risco de $15 \%$ de falso negativo. Conclui-se que não existe diferença clinicamente considerável entre um disco deslocado e um disco normal.
A ocorrência de sintomas têmporo-mandibulares em 168 adolescentes foi verificada por Loft et al. ${ }^{14}$, considerando a história de Ortodontia prévia ou não. Um questionário anamnésico contendo 18 perguntas foi respondido pelos adolescentes, e tanto os indivíduos do gênero feminino como os do gênero masculino foram tratados com aparelhos ortodônticos. Sintomas de dor e desconforto facial foram mais relatados por adolescentes do gênero feminino, os quais foram associados ao tratamento ortodôntico. Esses resultados alertam para a necessidade do ortodontista realizar um exame criterioso de sinais e sintomas de DTM antes de iniciar o tratamento ortodôntico.

Sidelsky e Clayton ${ }^{32}$ investigaram a presença dos sons da ATM. Concluíram que os sons da articulação têmporo-mandibular não representam um problema, quando em pacientes assintomáticos, e os ruídos na fase de abertura constituem-se em uma característica clínica questionável, quando não estão acompanhados de outros sintomas.

Zarb et al..$^{38}$ comentaram que a prevalência de sons originários da ATM ainda é objeto de discussão. Essa prevalência em pacientes assintomáticos está em torno de 15 a $65 \%$, sendo que $85 \%$ dos indivíduos normais produzem algum tipo de som articular em abertura máxima. Maiores estudos devem demonstrar a importância e a evidência dos sons articulares como diagnóstico diferencial de pacientes com disfunções da ATM.

Widmalm et $a 1 .{ }^{36}$, estudando 27 indivíduos autopsiados, demonstraram que os sons da ATM somente podem ser produzidos em articulações com discos ou superfícies anormais. Articulações normais eram consistentemente silenciosas. A presença de som articular é provavelmente um bom indicador de anormalidades articulares. A ausência de som articular não pode ser utilizada como regra da ausência de anormalidade articular. Sons como estalos ou crepitações parece não ser característica de diferentes tipos de patologias, como se pensava antigamente. 
O risco do tratamento ortodôntico, em relação às desordens têmporo-mandibulares, foi estudado através de uma revisão de literatura por Sado$w k^{30}{ }^{30}$. O resultado desta pesquisa foi que tratamentos ortodônticos, em crianças ou adolescentes, geralmente não são riscos para desenvolvimento de DTM no futuro. Essa conclusão foi obtida pelo autor, baseado em dois fatores: primeiro, existe uma multiplicidade de fatores que podem ser responsáveis por causar ou exacerbar as DTM; segundo, a mecanoterapia ortodôntica realiza mudanças graduais em um ambiente com grande capacidade adaptativa.

Ishigaki et al. ${ }^{10}$, após analisarem 355 pacientes que apresentavam dores faciais, sons articulares da ATM e limitação de abertura, concluíram que, somente com o exame da percepção dos sons, o clínico não é capaz de diagnosticar o tipo de desarranjo interno da articulação do paciente. Os autores chegaram a esta conclusão após constatarem que $40 \%$ dos pacientes da amostra estudada apresentavam deslocamentos do disco articular, porém sem presença de sons na ATM e assintomáticos.

Tallents et al. ${ }^{33}$ publicaram um artigo sobre sons articulares de 50 pacientes assintomáticos que não apresentavam sons à palpação. Os autores utilizaram gravação eletrônica dos sons articulares (vibrações), e concluíram que $44 \%$ de todas as articulações apresentavam sons identificáveis e que $50 \%$ dos sons eram emitidos quando o côndilo estava posicionado no ápice da eminência articular. Os sons sempre ocorriam no início da fase da abertura, sendo diagnosticados como normais pela ressonância magnética. Estes dados sugerem que eventos como ausência de sons articulares não demonstram o estágio de desarranjo interno da articulação têmporo-mandibular em pacientes assintomáticos.

O propósito do estudo de Tasaki et al. ${ }^{34}$ foi desenvolver um sistema de classificação e prevalência do deslocamento de disco articular, em pacientes sintomáticos e assintomáticos, baseado em imagens de ressonância magnética. Foram observados diferentes tipos de deslocamento do disco, unilateral ou bilateralmente em $82 \%$ dos pacientes sintomáticos e em $30 \%$ dos pacientes assintomáticos. Apesar da alta prevalência de deslocamento de disco em pacientes sintomáticos demonstrar estar associada à dor e disfunção têmporo-mandibular, sugere, também, que deslocamento de disco foi considerado comum em pacientes assintomáticos.

Ao estudarem a prevalência de desarranjos internos da articulação têmporo-mandibular em pacientes sintomáticos e assintomáticos, Katzberg et al. ${ }^{12}$ utilizaram imagens de ressonância magnética antes do tratamento ortodôntico. Os resultados da pesquisa demonstraram haver deslocamento do disco articular em 33\% dos pacientes assintomáticos, com diferença estísticamente significativa.

A prevalência de disfunção têmporo-mandibular foi avaliada por Morrant e Taylor ${ }^{20}$ em 301 pacientes pré-ortodônticos. Os pacientes responderam a um questionário padrão sobre sintomas de DTM e foram avaliados clinicamente quanto à presença de sinais. De acordo com os resultados, os sinais e sintomas de DTM encontrados não se correlacionaram com o gênero dos pacientes, porém os ruídos articulares acometeram os mais velhos, enquanto os indivíduos mais jovens apresentaram, freqüentemente, limitação de abertura bucal. Concluiu-se que, em um grande número de pacientes, uma condição sub-clínica de DTM está presente, podendo ser exacerbada com o tratamento ortodôntico. Baseado neste fato, os autores aconselharam a realização de um exame da ATM antes de se iniciar a terapia ortodôntica.

McNamara e Türp ${ }^{18}$ revisaram a relação do tratamento ortodôntico com as DTM e abordaram as seguintes questões: 1) Tratamento ortodôntico com aparelho fixo ou removível colabora para maior incidência de DTM? 2) Extrações de prémolares, como parte de um plano de tratamento ortodôntico, resultam em maior incidência de DTM? 3) Tratamento ortodôntico previne ou cura DTM? Baseados em pesquisas, encontraram 21 publicações, as quais respondiam às questões da 
investigação. Tratamento ortodôntico durante a adolescência não aumenta e nem diminui a chance de desenvolvimento de DTM no futuro. Não existe evidência de risco para DTM associado a qualquer tipo de mecânica ortodôntica. Extrações de dentes não aumentam o risco para DTM e não existe nenhuma evidência de que tratamento ortodôntico previne ou elimina DTM.

A relação entre o tratamento ortodôntico e as desordens têmporo-mandibulares (DTM), tem sido de grande interesse para os ortodontistas, mas somente na década passada, após um processo judicial que atribuiu a causa da DTM ao tratamento ortodôntico, houve um número significante de estudos clínicos e metodologicamente bem conduzidos que investigaram esta associação. Os resultados da pesquisas foram detalhados através de uma extensa revisão na literatura atual, por $\mathrm{McNa}$ mara $^{19}$, e resumidos do seguinte modo: 1) Sinais e sintomas de DTM podem ocorrer em pessoas saudáveis; 2) sinais e sintomas aumentam com a idade, particularmente, durante a adolescência, até a menopausa; a DTM que começa durante o tratamento ortodôntico não pode ser relacionada com o tratamento; 3) não existe risco para DTM associada em qualquer tipo de mecânica ortodôntica; 4) não existem evidências de que oclusão estável como meta de tratamento ortodôntico ideal previna sinais e sintomas de DTM; 5) a extração de dentes, como parte do plano de tratamento ortodôntico, não aumenta o risco para desenvolvimento de DTM. No entanto, existe pequena evidência de que tratamento ortodôntico previna DTM, na correção da mordida cruzada posterior unilateral em crianças.

Henrikson et al. ${ }^{7}$ estimaram a prevalência de sinais e sintomas de DTM, antes, durante e após o tratamento ortodôntico, em 65 adolescentes. A prevalência de sinais e sintomas foi medida com um questionário anamnésico e avaliação clínica em quatro períodos distintos: antes, durante, após o tratamento ortodôntico e um ano após a terceira avaliação. De acordo com os autores, o tratamen- to ortodôntico não aumentou os riscos de sinais e sintomas de DTM.

Garcia e Madeira ${ }^{4}$ estudaram os ruídos articulares em 34 pacientes portadores de desordens têmporo-mandibulares. Observaram, que o ruído articular é um dos sinais da DTM, que indica alterações na ATM, e permite estabelecer o diagnóstico clínico da patologia articular, mas a ausência de ruídos articulares não indica, necessariamente, uma ATM saudável, uma vez que o deslocamento do disco sem redução não provoca ruído.

O relacionamento entre tratamento ortodôntico e sinais e sintomas de desordens têmporomandibulares foi estudado por Henrikson e Nilner ${ }^{8}$, prospectiva e longitudinalmente, em 65 adolescentes do gênero feminino, com má oclusão de Classe 11 e tratamento ortodôntico, com ou sem extrações. Concluíram que o tratamento ortodôntico, com ou sem extrações, não aumenta e nem diminui os riscos para DTM, mesmo que o paciente tenha sinais e sintomas no pré-tratamento.

Nebbe, Major ${ }^{21}$ estudaram e avaliaram a prevalência do deslocamento do disco articular em adolescentes pré-ortodônticos (75 meninos e 119 meninas), através da imagem de ressonância magnética. Este estudo demonstra que, embora a prevalência de deslocamento de disco tenha sido mais alta em meninas do que em meninos, comprovou que este deslocamento ocorre freqüentemente em adolescentes pré-ortodônticos.

Pacientes assintomáticos que procuram tratamento ortodôntico deveriam ser avaliados e questionados, pelo ortodontista, a respeito de sinais e sintomas de DTM, antes de iniciar o plano de tratamento. Mao e Duan ${ }^{17}$, após pesquisas para estudar a atitude dos ortodontistas chineses, relacionando tratamento ortodôntico às DTM, através de um questionário, concluíram, que a maior parte dos ortodontistas pensa que o tratamento ortodôntico inadequado poderia aumentar o desenvolvimento de DTM e que o tratamento ortodôntico adequado poderia prevení-la. 


\section{DISCUSSÃO}

O controle das desordens têmporo-mandibulares, que podem ser apresentadas como dor orofacial, é um problema complexo em Odontologia. Essa complexidade surge devido às várias desordens que podem ser sintomáticas ou assintomáticas, no sistema mastigatório.

A suposta relação entre a Ortodontia e as disfunções têmporo-mandibulares tem despertado o interesse da classe ortodôntica nos últimos anos. Apesar dos significativos progressos na capacidade de diagnóstico por meio de técnicas avançadas como ressonância magnética, tomografia computadorizada 3D da ATM e a aplicação de procedimentos clínicos mais sofisticados, esses avanços não foram, porém, capazes de esclarecer a controvérsia sobre essa possível relação.

Um reflexo dessa controvérsia é a maneira como o tratamento ortodôntico é considerado nas diversas publicações. Se para alguns autores, a correção ortodôntica pode ser a cura das disfunções da ATM, para outros pode predispor o paciente a dores e disfunção do sistema estomatognático.

A fim de administrar efetivamente as desordens têmporo-mandibulares, o ortodontista deve ser capaz de diferenciá-las de qualquer outra condição de dor orofacial. Uma vez estabelecido o diagnóstico, o profissional pode, então, escolher a terapia apropriada.

Entre outros fatores, o exame clínico merece maior atenção por parte dos ortodontistas no diagnóstico e plano de tratamento em Ortodontia. A importância de se realizar um exame minucioso de sinais e sintomas, antes de iniciar o tratamento ortodôntico, é para que o paciente seja alertado quando da presença desses sinais e/ou sintomas ${ }^{37}$.

Alguns sinais e sintomas de DTM, que eventualmente apareçam durante o tratamento ortodôntico, podem não se associar diretamente a este, já que ocorre uma coincidência nas épocas da realização do tratamento e do surgimento dos mesmos ${ }^{18,19}$. A duração do tratamento também deve ser considerada, uma vez que esse tratamento pode se pro- longar por muitos anos, expondo, naturalmente, o paciente ao surgimento desses sintomas ${ }^{30}$.

Muitos adolescentes apresentam sintomas de DTM nesta fase em que estão sempre sendo assistidos pelo ortodontista, e que evidentemente os pacientes jovens possuem um alto limiar de tolerância, justificando a ausência de sintomatologia na maioria dos pacientes ${ }^{23}$. Mas estas disfunções podem não se manifestar durante os anos que o profissional mantém o paciente sob observação. No entanto, alguns destes jovens pacientes podem ter estes sintomas, e nunca mencioná-los ao ortodontista ${ }^{1}$.

Sugere-se que, em grande número de pacientes pré-ortodônticos, uma condição subclínica de DTM está presente, podendo ser exacerbada com o tratamento ${ }^{20}$. Por outro lado, pesquisas demonstram que, tratamento ortodôntico durante a adolescência não aumenta e nem diminui a chance de desenvolvimento de DTM no futuro, não é específico ou necessário para melhorar sinais e sintomas, $7,8,29,30 \mathrm{e}$ DTM que começa durante o tratamento não pode ser relacionada com o mesmo ${ }^{18,19}$.

Essa conclusão foi obtida baseada em dois fatores: primeiro, existe uma multiplicidade de fatores que podem ser responsáveis por causar ou exacerbar as DTM; segundo, a mecanoterapia ortodôntica realiza mudanças graduais em um ambiente com grande capacidade adaptativa ${ }^{30}$.

A maior parte dos ortodontistas pensa que o tratamento ortodôntico inadequado deveria aumentar o desenvolvimento de DTM e que tratamento adequado poderia prevení-la ${ }^{17}$. Mas não existem evidências de que oclusão estável previna sinais e sintomas de DTM ${ }^{19}$.

Confirmando a não associação do tratamento ortodôntico com sinais clínicos, através de um estudo comparativo entre pacientes tratados e não tratados ortodonticamente; avaliando a oclusão funcional com a função da ATM, encontraram bastante similaridade na prevalência de sinais e sintomas nas ATMs entre os grupos ${ }^{28}$.

Muito embora os sinais clínicos devam ser 
soberanos, ao se considerar a necessidade de tratamento ${ }^{35}$, índices baseados em relatos de sintomas podem orientar clínicos e pesquisadores na detecção desses problemas ${ }^{6}$. E quando se tenta comparar dados relativos à necessidade de tratamento de DTM, dificuldades são normalmente encontradas devido à falta de padronização de dados e, principalmente, na definição de sintomas considerados significativos ${ }^{10}$.

Outro aspecto extensamente discutido na literatura é a possível relação entre a presença de estalidos articulares e a Ortodontia. Muitos pacientes procuram por tratamento ortodôntico com o objetivo da eliminação desses ruídos.

De maneira oposta, o tratamento ortodôntico é considerado "responsável" pelo surgimento desses ruídos durante ou após o tratamento.

Esses estalidos normalmente estão associados à presença de deslocamento do disco articular para anterior ou antero-medial ${ }^{27,34}$. No passado, não muito distante, este era um fator considerado indicativo de tratamento ${ }^{11}$, atualmente admite-se que só se deve tratá-los quando associados à presença de dor e/ou disfunção ${ }^{32}$.

Um estudo realizado com 40 pacientes assintomáticos e clinicamente normais, demonstrou que $15 \%$ dos pacientes assintomáticos tinham alguma forma de deslocamento de disco que pode sugerir que um exame clínico negativo das articulações pode desenvolver um risco de $15 \%$ de falso negativo, comprovando não existir diferença clinicamente considerável entre um disco deslocado e um disco normal ${ }^{38}$.

Esse deslocamento de disco assintomático não provocava qualquer distúrbio funcional, como estalidos, movimentos irregulares ou limitação de abertura. No entanto, a ausência de ruídos na ATM não significa que ela se encontre bem posicionada, uma vez que o deslocamento do disco sem redução não provoca ruídos ${ }^{3,4,27,35}$. Mas também nem sempre a presença de sons é indicativa de patologias ${ }^{13}$.

O estalo, é o mais comum dos ruídos e repre- senta, um desequilíbrio mecânico da articulação têmporo-mandibular e dos músculos da mastigação $0^{25}$, deslocamento anterior de disco, o qual não indica necessariamente a existência de DTM $^{27}$. E os estalidos estão associados a irregularidades na eminência articular durante o percurso condilar'.

A prevalência de desarranjo interno de pacientes assintomáticos é significante, com valores em torno de $32 \%^{11,13}$. E a ausência de sons articulares não demonstra o estágio de desarranjo interno da ATM em pacientes assintomáticos, após constatarem que $40 \%$ da amostra estudada apresentavam deslocamentos do disco articular, porém sem a presença de sons na ATM e assintomáticos ${ }^{10,33}$. Portanto, o diagnóstico da imagem da ATM de um jovem paciente pré-ortodôntico deve ser cuidadosamente considerado no mínimo de resultados clínicos e interpretado para o benefício do paciente ${ }^{24}$.

Sons como estalos ou crepitação parecem não ser características de diferentes tipos de patologias, como se pensava antigamente ${ }^{3}$. No entanto a presença de som é provavelmente um bom indicador de anormalidades articulares ${ }^{35}$, mas a ausência de som não deve ser utilizada como fator indicativo de articulação normal ${ }^{3,27,35}$.

A prevalência de sons originários da ATM ainda é objeto de discussão $\mathrm{o}^{32,38}$. Esta prevalência em pacientes assintomáticos está em torno de 15 a $65 \%$, sendo que $85 \%$ dos indivíduos normais produzem algum tipo de som em abertura máxima.

Esse fato corrobora com achados de outros autores ${ }^{12,34}$ que, utilizando ressonância magnética, encontraram uma prevalência de 33\% de deslocamento de disco em pacientes assintomáticos e em $77 \%$ dos pacientes sintomáticos. Não foi possível correlacionar tratamento ortodôntico aos desarranjos internos da ATM, ou seja, o deslocamento do disco estava presente indistintamente nos pacientes com DTM e nos pacientes assintomáticos, tratados ou não ortodonticamente. Deslocamento de disco foi considerado comum em pacientes assintomáticos ${ }^{34}$. 
Porém, apesar da maior freqüência de disco deslocado em pacientes com DTM sintomáticos ${ }^{34}$, grande parcela de indivíduos assintomáticos apresenta essa alteração morfológica, não necessitando de tratamento. Para esses pacientes adolescentes, processos adaptativos de alteração tecidual de tecidos retrodiscais são responsáveis pela ausência de dor e disfunção ${ }^{26}$.

Deslocamento de disco pode ser associado com desenvolvimento de sintomas clínicos de dor craniofacial, uma vez que a capacidade adaptativa é reduzida quando o crescimento ativo está completo $^{21}$.

Parece uma boa conduta não adotar somente o exame clínico para concluir o diagnóstico das DTM, principalmente as assintomáticas ${ }^{10,14}$. Sendo assim, além do exame clínico, é aconselhável até que utilizemos exames complementares simples e de custos reduzidos, porém, que nos mantenham mais seguros do nosso diagnóstico em relação ao nosso paciente ${ }^{37}$.

Uma das justificativas para esta conduta, seria a grande prevalência das disfunções têmporo-mandibulares na população de um modo geral, e o fato de muitos profissionais associarem este termo às patologias que acometem apenas a articulação. No entanto, a disfunção inclui qualquer desarmonia que ocorra nas relações funcionais dos dentes e suas estruturas de suporte, dos maxilares, das articulações têmporo-mandibulares, dos músculos do aparelho estomatognático e dos suprimentos vascular e nervoso destes tecidos. O diagnóstico e tratamento das DTMs, devido à etiologia multifatorial das mesmas, tornam-se mais difíceis.

Portanto, antes de se iniciar o tratamento ortodôntico, o profissional deve diagnosticar e avaliar muito bem as DTM preexistentes, assim evita-se que um risco presumido esteja presente durante o tratamento ortodôntico.

\section{CONCLUSÕES}

Avaliando as informações obtidas na literatura estudada, conclui-se que:

1) A porcentagem de pacientes que apresentam DTM, assintomáticos, é significante, com valores em torno de $32 \%$.

2) A prevalência de ruídos articulares em pacientes assintomáticos está em torno de 15 a $65 \%$, sendo que $85 \%$ dos indivíduos normais produzem algum tipo de som.

2.1) Deslocamento de disco foi considerado um achado comum em pacientes assintomáticos.

2.2) A ausência de ruídos articulares não indica, necessariamente, uma ATM saudável, uma vez que deslocamento de disco sem redução não provoca ruídos.

3) O tratamento ortodôntico não aumenta e nem diminui os riscos para DTM, nem piora sinais e sintomas do pré-tratamento.

4) A literatura mostra-se concordante de que somente o exame clínico não indica todos os defeitos estruturais em pacientes pré-ortodônticos.

Enviado em: Fevereiro de 2004 Revisado e aceito: Novembro de 2004

\title{
Study of TMJ signs in orthodontic patients with no symptoms
}

\begin{abstract}
Study the presence of signs of temporomandibular desorders in asymptomatic patients in the beginning, during, and after the orthodontic treatment, to verify if the clinical examination is enough for a proper diagnosis, and if the orthodontic treatment is a factor that contributes for the development of temporomandibular dysfunction on this asymptomatic patients. The orthodontic treatment was not considered contributing factor for development of temporomandibular desorders.
\end{abstract}

Key words: Temporomandibular. Orthodontics. Corrective Orthodontics. Temporomandibular desorders. Occlusion. Malocclusion 


\section{REFERÊNCIAS}

1. AUBREY, R. B. Occlusal objectives in orthodontic treatment. Am J Orthod, St. Louis, v. 74, no. 2, p. 162,1978.

2. BENGSTON, A. L.; VONO, G. B.; TELLES, S. C. Oclusão e seus mistérios. In: RIBEIRO, S. C. Atualização clínica odontológica. 1. ed. São Paulo: Artes Médicas, 1992. cap.14, p.193-209.

3. ERIKSSON, L.; WESTESSON, P. L.; ROHLIN, M. Temporomandibular joint sounds in patients with disc displacement. Int J Oral Surg, Copenhagen, v.14, no. 5, p. 428-436,1985.

4. GARCIA, A. R.; MADEIRA, M. C. Ruídos articulares e o tratamento das desordens temporomandibulares. Rev Assoc Paul Cir Dent, São Paulo, v. 53, n. 2, p.109-15, mar./abr.1999.

5. GREENE, C. S. Orthodontics and temporomandibular disorders. Dent Clin North Am, Philadelphia, v. 32, no. 3, p. 529-538, July 1988.

6. HELKIMO, M. Epidemiological surveys of disfunction of the masticatory system. Oral Sci, v.7, p. 54-69, 1976.

7. HENRIKSON, T.; NILNER, M. Temporomandibular disorders and need of stomatognathic treatment in orthodontically treated and untreated girls. Eur J Orthod, London. No prelo 1999.

8. HENRIKSON, T.; NILNER, M.; KUROL, J. Signs of temporomandibular disorders, in girls receiving orthodontic treatment. A prospective and longitudinal comparison with untreated class II maloclusions and normal oclusion subjects. Eur J Orthod, London, v. 22, no. 3, p. 271-281, June 2000

9. ISBERG, A. M.; WESTESSON, P. L. Movement of disc and condyle in temporomandibular joints with and without clicking. Acta Odontal Scand, Stockholm, v. 40, p.165-177,1982.

10. ISHIGAKI, S.; BESSETE, R. W.; MARUYAMA, T. The distribution of internal deragement patients joint dysfuncion: prevalence, diagnosis and treatments. J Craniomandibular Pract, Chattanooga, v. 10, no. 4, p. 289-296, Oct. 1992.

11. KAPLAN, P. A. T. U. H. K.; WILLIAMS, S. M.; LYDIATT, D. D. The normal temporomandibular joint: $\mathrm{MR}$ and arthographi ccorrelation. Radiology, [Illinois?], v. 165, no. 1, p. 177-178, Oct. 1987.

12. KATZBERG, R. W.; WESTESSON, P. L.; TALLENTS, R. H.; DRAKE, C. M. Anatomic disorders of the temporomandibular joint disc assymptomatic subjects. J Oral Maxillofac Surg, Philadelphia, v. 54, p. $147-153,1996$.

13. KIRCOS, L. T.; ORTENDAHL, D. A.;MARK, A. S.; ARAKAWA, M. Magnectic resonance imaging of the TMJ disc in asymptomatic volunteers. J Oral Maxillofac Surg, Philadelphia, v. 45, no.10, p. 852-854, Oct. 1987

14. LOFT, G. H.; REYNOLDS, M. J.; ZWEMER, J. D.; THOMPSON, W. O. DUSHKU, J. The occurrence of craniomandibular symptoms in healthy young adults with and without prior orthodontic treatment. Am J Orthod Dentofacial Orthop, St. Louis, v. 96, no. 3, p. 264-265, Sept. 1989.

15. LUTHER, F. Orthodontics and the temporomandibular joint: where are we now ? Part 1. Orthodontic treatment and temporomandibular disorders. Angle Orthod, Appleton, v. 68, no. 4, p. 295-304, Aug. 1998a

16. LUTHER, F. Orthodontics and the temporomandibular joint: where are now? Part 2. Functional occlusion, malocclusion, and TMD. Angle Orthod, Appleton, v. 68, no. 4, p. 305-318, Aug. 1998b.

17. MAO, Y.; DUAN, X. H. Attitude of Chinese orthodontists towards the betweenorthodontic treatment and temporomandibular disorders. Int Dent J, [S. I.], v. 51, no. 4, p. 277-281, Aug. 2001.

18. McNAMARA, J. A ; TÜRP, J. C. Orthodontic treatment and temporomandibular disorders: is there a relationship? Part 1: clinical studies. J Orofac Orthop, München, v. 58, no. 2, p. 74-89,1997

19. McNAMARA, J. A. Orthodontic treatment and temporomandibular disorders. Oral Surg Oral Med Oral Pathol Oral Radiol Endod, St. Louis, v. 83, no.1, p.107-117, 1997

20. MORRANT, D. G.; TAYLOR, G. S. The prevalence of temporomandibular disorder in patients referred for orthodontic assessment. Br J Orthod, London, v. 23, no. 3, p. 261-265, Aug.1996.

21. NEBBE, B.; MAJOR, P. W. Prevalence of TMJ displacement in a pre orthodontic adolescent sample. Angle Orthod, Appleton, v. 70, no. 6, p. 454-463, May 2000.
22. PARKER, M. W. A dynamic model of etiology in temporomandibular disorders. J Am Dent Ass, Chicago, v. 120, p. 283-290,1990.

23. PERRY, H. T. Adolescent temporomandibular dysfuntion. Am J Orthod, St. Louis, v. 63, p. 517-525, 1973.

24. PETRIKOWSKI, C. G.; GRACE, M. G. A. Age and gender differencesin temporomandibular joint radiographic findings before orthodontic treatment in adolescents. J Oral Surg, Chicago, v. 87, p. $380-385,1999$

25. PULLINGER, A.G.; SELIGMAN, D. A. SOLBERG, W. K. Temporomandibulardesorders. II. Occlusal factors associated with temporomandibular join tenderness and dysfunction. J Prosthed Dent, St. Louis, v. 59, no. 3, p. 363-367, Mar. 1988.

26. RIBEIRO, R. F. The prevalence of disk displacement in symptomatic and assymptomatic volunteers aged 6 to 25 years. J Orofac Pain, Carol Stream, v. 11, p. 37-47,1997.

27. ROHLIN, M.; WESTESSON, P. L.; ERIKSSON, L. The correlation of temporomandibular joint sounds with joint morphology in fifty-five autopsy specimes. J Oral Maxillofac Surg, Philadelphia, v. 43, no. 3, p. 194-200, Mar.1995.

28. SADOWSKY, C.; BEGOLE, E. A. Long term status of temporomandibular joint function and functional occlusion after orthodontic treatment. Am J Orthod, St. Louis, v. 78, no. 2, p. 201-212, Aug. 1980.

29. SADOWSKY, C.; POLSON, A. M. Temporomandibular disorders and functional occlusion after orthodontic treatment : results two long - term studies. Am J Orthod Dentofacial Orthop, St. Louis, v. 86, no. 5, p. 386-390, Nov. 1984

30. SADOWSKY, C. The risk of orthodontic treatment for producing temporomandibular mandibular disorders: a literature overview. Am J Orthod Dentofacial Orthop, St. Louis, v. 101, no.1, p. 79-83, Jan. 1992.

31. SADOWSKY, C.; MUHL, Z. F.; SAKOLS, E. I.; SOMMERVILLE J. M. Temporomandibular joint sounds in orthodontic patients. J Dent Res, Chicago, v. 5, no. 6, p.193-207, Dec. 1985.

32. SIDELSKY, H.; CLAYTON, J. A. A clinical study of joint sounds in subjects with restored oclusions. J Prosthet Dent, St. Louis, v. 63, no. 5 , p. 580-586, May 1990.

33. TALLENTS, R. H.; HATALA, M.; KATZBERG, R. W.; WESTESSON, P. L. Temporomandibular joint sounds in asymptomatic volunteers. J Prosthet Dent, St. Louis, v. 69, no. 3, p. 298-304, Mar.1993.

34. TASAKI, M. M.; WESTESSON, P. L.; ISBERG, A. M.; REN, Y. F.; TALLENTS, R. H. Classification and prevalence of temporomandibular joint disc displacement in patients and sympton - free volunteers. Am J Orthod Dentofacial Orthop, St. Louis, v.109, p. 249-262,1996.

35. WESTESSON, P. L.; ERIKSSON, L.; KURITA, K. Relliabity of a negative clinical temporomandibular joint examination; prevalence of disc displacent in asymptomatic temporomandibular joints. Oral Surg Oral Med Oral Pathol, St. Louis, v. 68, no. 5, p. 551-554, Nov. 1989.

36. WIDMALM, S. E.; WESTESSON, P. L.; BROOKS, S. L.; HATALA, M. P.; PAESANI, D. Temporomandibular joint sounds: correlation to joint structure in fresh autopsy specimens. Acta Odontol Scand, Stockholm, v.101, no. 6, p. 60-69, June 1992.

37. WILLIANSON, E. H. The role of craniomandibular dysfunction in orthodonti diagnosis and treatment planning. Dent Clin North Am, Philadelphia, v. 27, no. 3, p. 541-560, July 1983.

38. ZARB, G.; LUND, J. P.; LAVIGNE, G.; FEINE, J. S.; GOLLET, J. P.; SHAYTOR, D. V.; SESSLE, B. J.; GREENWOOD, L. F.; HANNAM, A. G.; WOOD, W. W. The use of electronic devices in the diagnosis and treatment of temporomandibular disorders. J Can Dent Assoc, Ottawa, v. 56, p. 104-105, Feb. 1990.

\section{Endereço para correspondência}

Maria Eloiza Gaio Delboni

Rua 9 de julho - 1220,sl.101

Marília - SP

CEP: $17500-120$

E-mail: eloizadelboni@ig.com.br 